III. Ahr.

(Bodendorf-Altenburg.)

\begin{tabular}{|c|c|c|c|c|c|c|c|c|}
\hline \multirow[b]{2}{*}{ No. } & \multirow[b]{2}{*}{ Gemarkang } & \multirow[b]{2}{*}{ Bodenart } & \multirow[b]{2}{*}{ Traubensorte } & \multirow{2}{*}{ 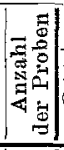 } & \multicolumn{2}{|c|}{ Schwankungen } & \multicolumn{2}{|c|}{ Mittel } \\
\hline & & & & & $\begin{array}{l}\text { Mostgewicht } \\
\text { (Grade nach } \\
\text { oechs le) }\end{array}$ & $\begin{array}{c}\text { Säure } \\
\% \\
\end{array}$ & \begin{tabular}{|c|} 
Mostge- \\
wicht \\
(Grade \\
Oeechslei
\end{tabular} & $\begin{array}{c}\text { Säure } \\
\%\end{array}$ \\
\hline 76 & Bodendorf & Schiefer, Stein, Lehm & Spätburgunder & 6 & $51,8-80,8$ & $1,02-1,61$ & 71,3 & 1,24 \\
\hline 77 & Heimersheim & Schiefer & $\begin{array}{l}\text { Spätburgunder, } \\
\text { Rlesling }\end{array}$ & 2 & $77,0 \cap .96,8$ & 1,00 u. 1,57 & 86,9 & 1,28 \\
\hline 78 & Nenenahr & Schiefer & Spätburgunder & 1 & 71,2 & 1,32 & - & - \\
\hline 79 & Ahrwerler & $\begin{array}{l}\text { Schiefer, Lehm, Kies, Ton- } \\
\text { stbjefer, Sandstein }\end{array}$ & $\begin{array}{l}\text { Spätburgunder, } \\
\text { Portugieser, Bur- } \\
\text { gunder }\end{array}$ & 18 & $63,3-93,6$ & $0,90-1,45$ & 76,6 & 1,19 \\
\hline 80 & Walporzheim & Schiefer, Kies, Lehm & $\begin{array}{l}\text { Spätburgunder, } \\
\text { Kleinberger, Ort- } \\
\text { lieber, Burgunder }\end{array}$ & 8 & $46,5-88,5$ & $1,06-1,71$ & 69,9 & 1,37 \\
\hline 81 & Dernau & Schiefer, Mergel, Lehm & $\begin{array}{l}\text { Spätburgunder, } \\
\text { Portrgieser }\end{array}$ & 18 & $66,5-81,9$ & $0,96-1,50$ & 72,3 & 1,28 \\
\hline 82 & Maysehoss & Scbiefer, Lehm & Spätburgunder & 14 & $60,9--77,1$ & $1,26-1,80$ & 68,4 & 1,43 \\
\hline 83 & Rech & Sehiefer, Lehm, Mergel, Kiesel & $\begin{array}{l}\text { Spätburgunder, } \\
\text { Kleinherger, Portu- } \\
\text { gieser }\end{array}$ & 15 & $55,0-79,7$ & $1,20-1,96$ & 67,2 & 1,49 \\
\hline 84 & Reimerzhoven & Schiefer & Ortlieber & 1) & 50,8 & 1,82 & - & - \\
\hline 85 & Altenahr & Schiefer, Lehm & $\begin{array}{l}\text { Sputiburgunder, } \\
\text { Plesing }\end{array}$ & 11 & $43,6-73,0$ & $\mid 1,26-1,74$ & 63,6 & 1,51 \\
\hline \multirow[t]{2}{*}{86} & Altenburg & Schiefer & Spätburgunder & $1]$ & 62,0 & 1,85 & - & - \\
\hline & & & Gesamt-Mittel & 95 & 一 & - & $\mathbf{7 0 , 4}$ & 1,36 \\
\hline
\end{tabular}

\title{
Moste des Jahrganges 1912 ans dem Gebiete der Mosel und ihrer Nebenflüsse.
}

Von

\section{A. Wellenstein.}

Mitteilungen aus dem Nahrungsmittel-Untersuchungamt der Stadt Trier.

[Eingegangen am 8. Juni 1913.]

Wenn man im verflossenen Herbste zur Zeit der Traubenlese die Gemarkungen unseres Weinbaugebietes durchstreifte, so bot sich dem Beschauer ein trauriges Bild der Verwästung dar. Braungefärbt und teilweise eingeschrumpft hingen die Beeren an den blattlosen Rebstöeken. Die berechtigten frohen Hoffnungen unserer Winzer auf eine quantitativ sehr reiche und qualitativ mittlere Ernte, welche den Weinbauern ermöglicht hätte, die durch die Fehljahre 1909 und 1910 hervorgerufenen wirtschaftlichen Schädigungen auszugleichen, hatte das Werk zweier Frostnächte vernichtet.

Der durch den Frost entstandene Schaden wies allerdings in den einzelnen Gemarkungen des Regierungsbezirks Trier einen verschiedenen Umfang anf. Am schwersten sind die Gebiete betroffen worden, deren Trauben noch nicht ,im Wein" waren, d. h. den nötigen Reifezustand noch nicht erreicht hatten. Es kommen hier vornehmlich in Frage das Weinbaugebiet der Obermosel, besonders aber das der Saar. Die Mittel- and Untermosel, wo die Traubenreife bereits ziemlich weit vor geschritten war, hatten weniger schwer unter dem Frost zu leiden. Trotzdem beläuft sich der durch die Frühfröste angerichtete Schaden, von welchem das gesamte Gebiet der Mosel und ihrer Nebenflüsse betroffen worden ist, auf viele Millionen Mark. 
Die Lese der durch den Frost geschädigten Trauben setzte an der Obermosel sofort nach Eintritt des Frostes ein. In den übrigen Gegenden unseres Weinbaugebietes suchte man durch längeres Hängenlassen der Trauben den gefürchteten Frostgeschmack des Mostes bezw. des Weines, allerdings auf Kosten der Mostmenge, fernzuhalten.

Während man noch vor Eintritt des Frostes in dem gesamten Moselweinbaugebiet auf einen nahezu ,vollen Herbst" rechnete, ist durch den Frost, abgesehen von der qualitativen Wertverminderung, eine Verminderung der Erntemenge bis auf $1 / 3$, in einzelnen Gegenden (Obermosel und Saar) sogar bis auf $1 / 5$ des erwarteten Ernteertrages eingetreten.

Der Preis dieser durch den Frost geschädigten Moste und Jungweine war ein auberordentlich niedriger; an der Obermosel und Saar betrug er nur etwa $300-400 \mathrm{Mk}$. für 1000 Liter. An der Mittel- und Untermosel, wo die Frostschäden nicht den Umfang erreichten wie in den übrigen Teilen des Weinbaugebietes, war er durchwegs höher, hielt sich jedoch immer noch erheblich unter dem Durchschnitt mittelguter Jahre.

Nach ihrer chemischen Zusammensetzung sind die 1912-er Moste des Moselweinbaugebietes, wie aus nachstehenden Tabellen hervorgeht, gekennzeichnet durch hohe Säure und niedrige Mostgewichte.

In den Annalen des Moselweinbaues wird das Jahr 1912 als ein gänzliches Fehljabr verzeichnet werden müssen.

a) Sa ar.

\begin{tabular}{|c|c|c|c|c|c|c|c|c|c|}
\hline \multirow[b]{2}{*}{ No. } & \multirow[b]{2}{*}{ Gomarkung } & & \multirow[b]{2}{*}{ Bodenart } & \multirow[b]{2}{*}{ Traubensorte } & \multirow{2}{*}{ 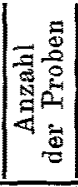 } & \multicolumn{2}{|c|}{ Schwankungen } & \multicolumn{2}{|c|}{ Mittel } \\
\hline & & & & & & $\begin{array}{c}\text { Most- } \\
\text { geWicht } \\
\text { (Grade naah } \\
\text { Oeehsio) }\end{array}$ & $\begin{array}{c}\text { Säure } \\
\% / \omega 0\end{array}$ & 变总 & 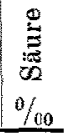 \\
\hline 1 & Merzig , . & & Ackerboden & Riesling Kleinb. & 2 & 41 u. 47 & 13,6 u. 16,9 & 44 & 15,3 \\
\hline 2 & Serrig . & & Schiefer & Riesling & 3 & $40-46,6$ & $23,9-27,7$ & 44,2 & 25,9 \\
\hline 3 & Saarburg . & & , & $"$ & 24 & $40-62,2$ & $13,6-29,4$ & 50,9 & 21,1 \\
\hline 4 & Beurig. . & & , & $"$ & 1 & 52 & 17,6 & & \\
\hline 5 & Irsch. . . & & $"$ & $"$ & 6 & $52,3-56,7$ & $15,5-20,9$ & 54,5 & 18,3 \\
\hline 6 & Niederleuken & & $"$ & 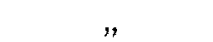 & 7 & $53-60,7$ & $15,3-19,2$ & 43,1 & 16,6 \\
\hline 7 & Ockfen & & $"$ & $"$ & 10 & $48-59,1$ & $17-28,7$ & 53,1 & 20,1 \\
\hline 8 & Ayl . . . & & ", & " & 7 & $40-58$ & $14,3-22,6$ & 51,2 & 17,6 \\
\hline 9 & Biebelhausen & & , & $"$ & 2 & $56,2 u .57$ & $15 \mathrm{u}, 15,5$ & 56,6 & 15,3 \\
\hline 10 & Schoden. . & & $"$ & $n$ & 6 & $54-58$ & $15,6-19,1$ & 55,3 & 17,1 \\
\hline 11 & Wiltingen . & & $"$ & $"$ & 11 & $48,5-65,6$ & $15,4-20,8$ & 55 & 17 \\
\hline 12 & Oberemmel & & $"$ & , & 10 & $43-52,7$ & $15,5-20,3$ & 48 & 18,4 \\
\hline 13 & Pellingen. & & Schiefer, Kalk & " & 5 & $|32,5-39,6|$ & $19,5-22,5$ & 37,5 & 20,8 \\
\hline 14 & Franzenheim & & Schiefer & " & 3 & $|42,8-46,4|$ & $23-26,5$ & 44,8 & 24,8 \\
\hline 15 & Hockweiler & & $"$ & $"$ & 1 & 38,5 & 26,4 & & \\
\hline 16 & Crettnach. & & $"$ & $n$ & 10 & $37,5-49,1$ & $14,3-23,6$ & 42 & 18,3 \\
\hline 17 & Commlingen & & $"$ & $"$ & 5 & $40-49,5$ & $14-22,6$ & 45,9 & 18,5 \\
\hline 18 & Canzem. & & $n$ & , & 6 & $53,5-66$ & $13,1-16,5$ & 61,8 & 15,1 \\
\hline 19 & Wawern. & & $"$ & $n$ & 10 & $52-73,2$ & $12-14$ & 56,7 & 12,6 \\
\hline 20 & Filzen . & & $"$ & $"$ & 4 & $55,6-61,9 \mid$ & $12,8-14,2$ & 58 & 13,4 \\
\hline 21 & Coenen & & , & $"$ & 3 & $50--57$ & $|11,8-14,1|$ & 54,5 & 13,2 \\
\hline 22 & Niedermennig & & $"$ & $"$ & 5 & $41-53,6$ & $\mid 15,9-21,7$ & 44,8 & 17,9 \\
\hline & & & & & 141 & Gesa & mt-Mittel & 50,4 & 18,3 \\
\hline
\end{tabular}


b) Obermosel und Sauer.

\begin{tabular}{|c|c|c|c|c|c|c|c|c|c|c|}
\hline \multirow[b]{2}{*}{ No. } & \multirow{2}{*}{\multicolumn{3}{|c|}{ Gemarkung }} & \multirow[b]{2}{*}{ Bodenart } & \multirow[b]{2}{*}{ Traubensorte } & \multirow{2}{*}{ 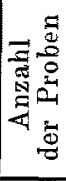 } & \multicolumn{2}{|c|}{ Schwankungen } & \multicolumn{2}{|c|}{ Mittel } \\
\hline & & & & & & & $\begin{array}{c}\text { Most- } \\
\text { gewitht } \\
\text { (Grade naech } \\
\text { Oechsle) }\end{array}$ & $\begin{array}{c}\text { Säure } \\
\% \\
\end{array}$ & $\frac{1}{30}$ & $\begin{array}{l}\stackrel{0}{\Xi} \\
0 \\
0 \\
0\end{array}$ \\
\hline 23 & Perl & & & Kalk & Kleinberg & 3 & $51,4-57,4$ & $15,3-16,4$ & 54,4 & 15,7 \\
\hline 24 & Sehndorf & & & " & " & 3 & $43,1-46,2$ & $15-15,5$ & 44,8 & 15,3 \\
\hline 25 & Nennig . & & & toniger Lehm & ", & 17 & $44,5-58,4$ & $14,8-20,4$ & 52,4 & 17,4 \\
\hline 26 & Kreuzweiler & & & Kalk & " & 5 & $48,1--56$ & $13,3-16,9$ & 51,4 & 15,6 \\
\hline 27 & Palzem . & . & & $"$ & , & 7 & $46-66,5$ & $15,3-19,6$ & 54,9 & 16,9 \\
\hline 28 & Wehr : & . & & " & $"$ & 8 & $37,5-62,5$ & $14,8-10,2$ & 58,3 & 16,1 \\
\hline 29 & Helfant. . & . & & , & , & 15 & $40-54$ & $14,5-19$ & 49 & 16,8 \\
\hline 30 & Wincheringen & . & . & $"$ & $\begin{array}{l}\text { Kleinberg } \\
\text { Sylvaner }\end{array}$ & 15 & $39-55,5$ & $15,6-22,4$ & 48,1 & 18,3 \\
\hline 31 & Rehlingen & . & & ", & Kleinberg & 2 & $43 u .55$ & 16,5 u. 21,7 & 49 & 19,1 \\
\hline 32 & Nittel . & & . & , &, & 16 & $40-64,5$ & $16-27,7$ & 53.3 & 19,3 \\
\hline 33 & Onsdorf & & & Kalk, Kalkboden & $n$ & 5 & $29-42$ & $20-26,6$ & 36,9 & 23,3 \\
\hline 34 & Wellen & & & Kalk & , & 9 & $42-68,5$ & $16.4-24$ & 52,8 & 19,8 \\
\hline 35 & Fellerich & . & & $"$ &, & 3 & $39-51$ & $22,2-24,3$ & 45,5 & 23,2 \\
\hline 36 & Tawern. & . & & , & $"$ & 2 & 43 u. 52 & $14,7 \cup 19,4$ & 47,5 & 17,1 \\
\hline 37 & Temmels & & & Kalk, Kalkboden & 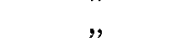 & 11 & $42-55,2$ & $16,8-23,3$ & 49 & 20,2 \\
\hline 38 & Oberbillig . & . & . & Kalk & $n$ & 13 & $43-61$ & $15,2-26,5$ & 46,4 & 18 \\
\hline 39 & Liersberg. & & 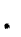 & $"$ & " & 2 & $50,2 \mathrm{u} \cdot 61$ & $12 \mathrm{u} .17,3$ & 55,6 & 14,7 \\
\hline 40 & Wasserliesch & & & Kalk & Kleinberg & 3 & $46,5-53$ & $17-22,5$ & 49,2 & 19,9 \\
\hline 41 & IgeI . . & & & Kalk, Gips & $\eta$ & 5 & $46-52$ & $14,4-18,1$ & 49,8 & 16 \\
\hline 42 & Metzdorf & & & Kalk & $n$ & 9 & $42--53$ & $15,6-20,7$ & 42,9 & 17,9 \\
\hline 43 & Grevenich . & & & $"$ & $"$ & 2 & 55 u. 57 & 14,3 u. 15 & 56 & 14,7 \\
\hline 44 & Mesenich & & & $"$ & $"$ & 6 & $46-57$ & $12,4-16,8$ & 51,5 & 15,2 \\
\hline \multirow[t]{2}{*}{45} & Langsur. . & & & $"$ & $n$ & 24 & $39,4-68$ & $10,9-22,3$ & 57,2 & 16,7 \\
\hline & & & & & & 185 & \multicolumn{2}{|c|}{ Gesamt-Mittel } & 50,9 & 17,8 \\
\hline
\end{tabular}

c) Mittelmosel

(von Conz bis 'Trittenheim).

\begin{tabular}{|c|c|c|c|c|c|c|c|c|c|}
\hline 46 & Conz. & . & Schiefer, Lehm & Riesl. Kleinbg. & 8 & $53-55,5$ & $8,6-17,7$ & 48 & \\
\hline 47 & Feyen . . & . & & $"$ & 2 & $52 \mathrm{u} .52,3$ & 14,5 u. 21 & 52,2 & 17 \\
\hline & St. Matthias & . & Schiefer & & 4 & $46,7-59$ & $14,2-18,8$ & 53,1 & \\
\hline & Trsch . . . & . & $"$ & Riesling & 1 & 55 & 13 & & \\
\hline & Kernscheid. & * & $"$ & , & 1 & 59 & 16,8 & & \\
\hline & Olewig . . & . & $"$ & Riesl. Sylvaner & 5 & $50-56,8$ & $12-17,1$ & 53,9 & \\
\hline & Trier. . & • & Schiefer, Luhm & $\begin{array}{l}\text { Riesl. Kleinberg } \\
\text { Oesterr., Sylvan. }\end{array}$ & 9 & $40,6-70$ & $12,5-19,8$ & 55,3 & \\
\hline & Tarforst & & Schiefer & Riesling & & 38,5 & 23 & & \\
\hline & Kürenz . & . & Schiofer, Lehm & & 1 & 49,2 & 13,3 & & \\
\hline & Kenn. & . & $\begin{array}{l}\text { schwerer Ton- } \\
\text { schiefer }\end{array}$ & $\begin{array}{l}\text { Riesling } \\
\text { Kleinberg }\end{array}$ & 5 & $46,5-61$ & $16,3-24,6$ & 54,4 & \\
\hline
\end{tabular}




\begin{tabular}{|c|c|c|c|c|c|c|c|c|c|}
\hline \multirow[b]{2}{*}{ No. } & \multirow{2}{*}{\multicolumn{2}{|c|}{ Gemarkung }} & \multirow[b]{2}{*}{ Bodenart } & \multirow[b]{2}{*}{ Traubensorte } & \multirow{2}{*}{ 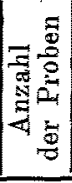 } & \multicolumn{2}{|c|}{ Schwankungen } & \multicolumn{2}{|c|}{ Mittel } \\
\hline & & & & & & $\begin{array}{c}\text { Most- } \\
\text { gewieht } \\
\left(\begin{array}{c}\text { Grade nach } \\
\text { Oechsle }\end{array}\right.\end{array}$ & $\begin{array}{c}\text { Säure } \\
\% \\
\% 00\end{array}$ & 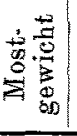 & 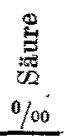 \\
\hline 56 & Schweich & - & $\begin{array}{l}\text { Schieferbod., leh- } \\
\text { miger Schiefer }\end{array}$ & Riesl. Kleinbg. & 13 & $51,2-73,8$ & $\mid 1,1-15,1$ & 62,2 & 12,7 \\
\hline 57 & Longuich & . & $\begin{array}{l}\text { Schiefer, lehmi- } \\
\text { ger Schiefer }\end{array}$ & $"$ & 19 & $45-75$ & $|12,4-18,5|$ & 63,2 & 14,5 \\
\hline 58 & Fastrau . & . & Schiefer & $"$ & 7 & $29,1-77,2$ & $8,5-15,1$ & 52,8 & 12,8 \\
\hline 59 & Longen . & . & $"$ & $"$ & 11 & $60,5-71$ & $12,3-15,5 \mid$ & 65,4 & 13,4 \\
\hline 60 & Lörsch . & . & $"$ & $"$ & $18-$ & $59-73,1$ & $9,3-17,3$ & 66,9 & 12,9 \\
\hline 61 & Fell . . & . & $"$ & Riesling & 13 & $50-61$ & $13,6-20,6$ & 57,4 & 16,4 \\
\hline 62 & Mehring . & $\cdot$ & $\begin{array}{l}\text { Schiefer, lehmig. } \\
\text { Schiefer, Boden }\end{array}$ & Kleinberg & 17 & $65-77,8$ & $|10,7-15,6|$ & 71,1 & 13,5 \\
\hline 63 & Pölich . & 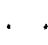 & Schiefer & Riesling & 5 & $64-81,7$ & $13,3-15$ & 37,5 & 14 \\
\hline 64 & Schleich. & 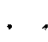 & Schiefer, Boden & Riesl. Kleinberg & 8 & $49-60$ & $14,4-17,7$ & 57,3 & 16,2 \\
\hline 65 & Detzem . & . & Lehm & $"$ & 10 & $54-69$ & $12,8-17,1$ & 60,8 & 14,8 \\
\hline 66 & Enseh . & - & Schiefer, Boden & $"$ & 11 & $49-65,5$ & $\mid 13-16,8$ & 55,5 & 14,9 \\
\hline 67 & Thörnich & & Schiefer & $"$ & 9 & $65-80$ & $11,3-14,5$ & 73,9 & 12,8 \\
\hline 68 & Becond . & • & Schiefer, Boden & $"$ & 10 & $50-66$ & $9,7-15$ & 56,4 & 12,5 \\
\hline 69 & Clusserath & 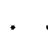 & " & $"$ & 37 & $52,5-80$ & $9-14,6$ & 65,9 & 12,4 \\
\hline 70 & Köwerich . & $\cdot$ & $"$ & $"$ & 10 & $59-70$ & $14,2-16,2$ & 65,3 & 15 \\
\hline 71 & Leiwen . & . & & $n$ & 11 & $50-61,5$ & $14,5-22,1$ & 56,8 & 18,2 \\
\hline \multirow[t]{2}{*}{72} & \multirow[t]{2}{*}{ Trittenheim } & & \multirow{2}{*}{$\begin{array}{l}\text { Schiefer, Boden } \\
\text { Kies }\end{array}$} & \multirow[t]{2}{*}{$"$} & 19 & $57-69$ & $\mid 12,7-16$ & 63,1 & 14,3 \\
\hline & & & & & 260 & $\mathrm{Ges}$ & mt-Mittel & 61,8 & 14,3 \\
\hline
\end{tabular}

d) Mittelmosel

(von Neumagen bis Reil).

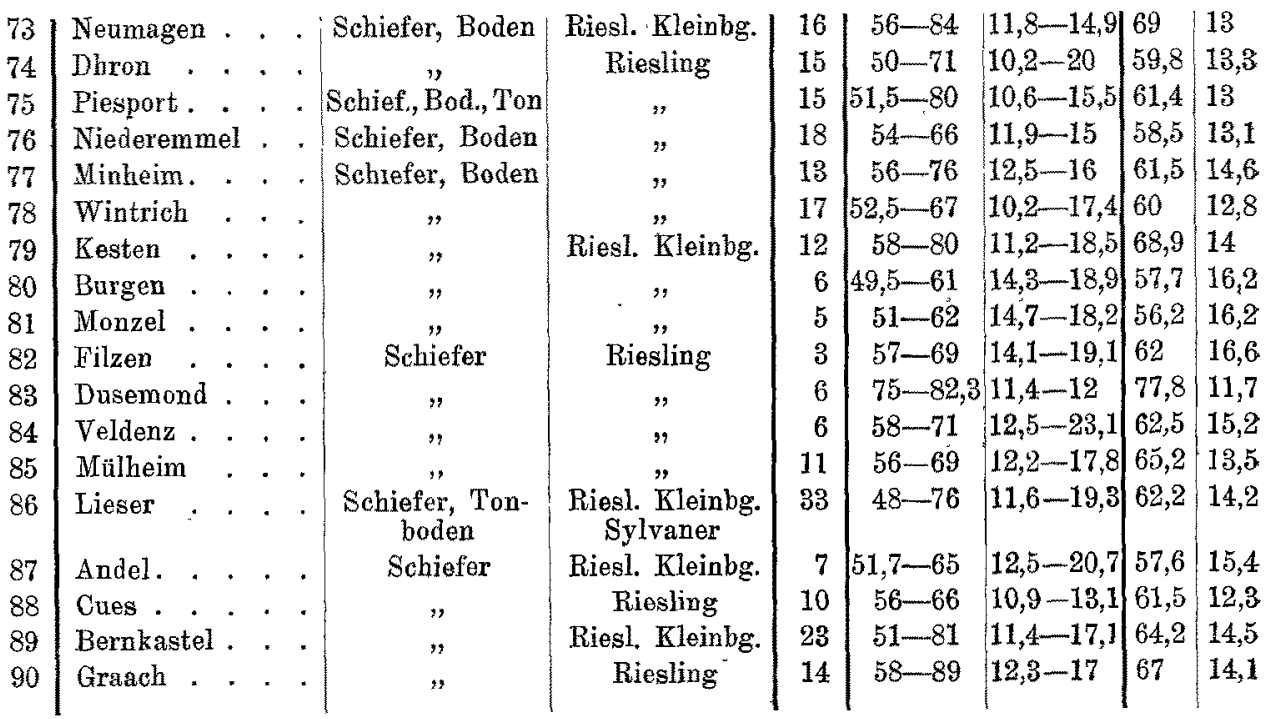




\begin{tabular}{|c|c|c|c|c|c|c|c|c|c|}
\hline \multirow[b]{2}{*}{ No. } & \multirow{2}{*}{\multicolumn{2}{|c|}{ Gemarkung }} & \multirow[b]{2}{*}{ Bodenart } & \multirow[b]{2}{*}{ Traubensorte } & \multirow{2}{*}{ 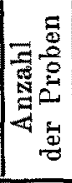 } & \multicolumn{2}{|c|}{ Schwankungen } & \multicolumn{2}{|c|}{ Mittel } \\
\hline & & & & & & $\begin{array}{c}\text { Most- } \\
\text { gew tcht } \\
\text { (Grade nach } \\
\text { (Oeehsle }\end{array}$ & $\begin{array}{c}\text { Säure } \\
\% \\
\% 00\end{array}$ & 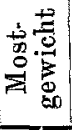 & 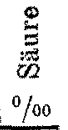 \\
\hline 91 & Wehlen. & & Schiefer & Riesling & 28 & $49-85,2$ & $10,6-17$ & 62,4 & 18,1 \\
\hline 92 & Zeltıngen . & & , & $n$ & 18 & $55-84,5$ & $9,8-14,7$ & 64,3 & 13,2 \\
\hline 93 & Rachtig. . & & $"$ & 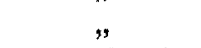 & 19 & $34,5-69,2$ & $10-15,9$ & 55,7 & 14 \\
\hline 94 & Uerzig . . & & $"$ & Ries1. Kleinbg. & 14 & $61-78,5$ & $\mid 13-17,4$ & 70,7 & 15,5 \\
\hline 95 & Bausendorf. & & , & " & 3 & $43,3-55$ & $10,3-19,4$ & 50,5 & 13,5 \\
\hline 96 & Erden. & & " & Riesling & 16 & $59-83$ & $10,6-15,4$ & 73,3 & 13,7 \\
\hline 97 & Lösenich. & & , & , & 10 & $44,6-81,5$ & $10,2-20,5$ & 71,5 & 14,1 \\
\hline 98 & Kinheim . & & $"$ & $"$ & 14 & $53,7-81$ & $11,8-15,1$ & 69,2 & 13,4 \\
\hline 99 & Crör. & & $"$ & , & 31 & $53-84$ & $10-17,9$ & 62,9 & 13,9 \\
\hline 100 & Wolf. . & & $"$ & $"$ & 6 & $55,2-71,8$ & $13,1-18,2$ & 63,3 & 15,4 \\
\hline 101 & Kövenich & . & $n$ & $n$ & 8 & $46-61$ & $18,2-22,4$ & 52,8 & 20,4 \\
\hline \multirow[t]{2}{*}{102} & \multirow[t]{2}{*}{ Reil . . . } & & \multirow[t]{2}{*}{$"$} & \multirow[t]{2}{*}{$n$} & 18 & $47-67$ & $19,2-21$ & 59,5 & 16,6 \\
\hline & & & & & 415 & Gesa & amt-Mittel & 63 & $\overline{14,1}$ \\
\hline
\end{tabular}

e) Ruwer.

\begin{tabular}{c|l|c|}
103 & Morscheid . . . & Schiefer \\
104 & Riveris . . . . & $"$ \\
105 & Gusterath . . . & $"$ \\
106 & Waldrach . . . & $"$ \\
107 & Casel . . . . . & $"$ \\
108 & Mertesdorf. . . & $"$ \\
109 & Eitelsbach . . . & Schiefer, Lehm \\
110 & Ruwer. . . . & $"$
\end{tabular}

\begin{tabular}{|c|c|c|c|c|c|}
\hline Riesling & & & 15.6 & & \\
\hline 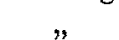 & 5 & $35-60,9$ & $25-29,7$ & 53.4 & 27.2 \\
\hline "* & 1 & 44 & 29,3 & & \\
\hline & 6 & $56,2-64$ & $12,2-15$ & 59,9 & 13,6 \\
\hline$"$ & 10 & $38,5-67,5$ & $11,5-25$ & 55,3 & 16,2 \\
\hline$"$ & 5 & $52-60$ & $\mid 11,3-17,6$ & 55,8 & 13,3 \\
\hline$"$ & 2 & 42,1 a. 60 & $12,1 u, 21,5$ & 51,1 & 16,8 \\
\hline$"$ & 9 & $42-60.4$ & $|11,4-22,9|$ & 53,4 & $13, \tilde{5}$ \\
\hline & 39 & Ges & at-Mittal & 54,9 & 16,5 \\
\hline
\end{tabular}

f) Lieser.

\begin{tabular}{l|l|c}
111 & Hupperath . . . & Schiefer \\
112 & Wittlich. . . . & $"$ \\
113 & Platten . . . . & $"$ \\
114 & Osann . . . . & $"$ \\
115 & Maring.Noviand . & $"$ \\
116 & Siebenborn . . . & $"$
\end{tabular}

\begin{tabular}{|c|r|c|c|c|c} 
Riesl. Kleinberg & 1 & 55 & 17 & & \\
", & 7 & $51,2-67$ & $14,8-19$ & 58,5 & 16,2 \\
Riesling & 5 & $57-71$ & $11,9-17,1$ & 61,8 & 15,3 \\
Riesl. Kleinberg & 24 & $47,2-60$ & $\mathbf{1 2 , 8 - 2 2 , 5}$ & 55,7 & 16,7 \\
Riesling & 19 & $40-64,3$ & $10,8-20,1$ & 57,4 & 15,5 \\
$" \prime$ & 1 & 52,5 & 21,4 & & \\
\hline & 57 & \multicolumn{2}{|c|}{ Gesamt-Mittel } & $\mathbf{5 7 , 1}$ & $\mathbf{1 6 , 2}$
\end{tabular}

